\title{
Automated Termination Proofs for Java Programs with Cyclic Data ${ }^{\star}$
}

\author{
Marc Brockschmidt, Richard Musiol, Carsten Otto, and Jürgen Giesl \\ LuFG Informatik 2, RWTH Aachen University, Germany
}

\begin{abstract}
In earlier work, we developed a technique to prove termination of Java programs automatically: first, Java programs are automatically transformed to term rewrite systems (TRSs) and then, existing methods and tools are used to prove termination of the resulting TRSs. In this paper, we extend our technique in order to prove termination of algorithms on cyclic data such as cyclic lists or graphs automatically. We implemented our technique in the tool AProVE and performed extensive experiments to evaluate its practical applicability.
\end{abstract}

\section{Introduction}

Techniques to prove termination automatically are essential in program verification. While approaches and tools for automated termination analysis of term rewrite systems (TRSs) and of logic programs have been studied for decades, in the last years the focus has shifted toward imperative languages like $C$ or Java.

Most techniques for imperative languages prove termination by synthesizing ranking functions (e.g., [12 26]) and localize the termination test using Ramsey's theorem 23 27. Such techniques are for instance used in the tools Terminator 413] and LoopFrog 22 31] to analyze termination of C programs. To handle the heap, one can use an abstraction [14] to integers based on separation logic [24].

On the other hand, there also exist transformational approaches which automatically transform imperative programs to TRSs or to logic programs. They allow to re-use the existing techniques and tools from term rewriting or logic programming also for imperative programs. In [17, C is analyzed by a transformation to TRSs and the tools Julia [30] and COSTA [2] prove termination of Java via a transformation to constraint logic programs. To deal with the heap, they also use an abstraction to integers and represent objects by their path length.

In 6 178 825 we presented an alternative approach for termination of Java via a transformation to TRSs. Like [230], we consider Java Bytecode (JBC) to avoid dealing with all language constructs of Java. This is no restriction, since Java compilers automatically translate Java to JBC. Indeed, our implementation handles the Java Bytecode produced by Oracle's standard compiler. In contrast to other approaches, we do not treat the heap by an abstraction to integers, but by an abstraction to terms. So for any class $\mathrm{Cl}$ with $n$ non-static fields, we use an $n$-ary function symbol $\mathrm{Cl}$. For example, consider a class List with two fields value and next. Then every List object is encoded as a term List $(v, n)$ where

\footnotetext{
^ Supported by the DFG grant GI 274/5-3.
} 
$v$ is the value of the current element and $n$ is the encoding of the next element. Hence, a list "[1,2]" is encoded by the term $\operatorname{List}(1, \operatorname{List}(2$, null $))$. In this way, our encoding maintains much more information from the original program than a (fixed) abstraction to integers. Now the advantage is that for any algorithm, existing tools from term rewriting can automatically search for (possibly different) suitable well-founded orders comparing arbitrary forms of terms. For more information on techniques for termination analysis of term rewriting, see, e.g., 16 20 33. As shown in the annual International Termination Competition 1 due to this flexibility, the implementation of our approach in the tool AProVE [19] is currently the most powerful termination prover for Java.

In this paper, we extend our technique to handle algorithms whose termination depends on cyclic objects (e.g., lists like "[0,1,2,1,2,..." or cyclic graphs). Up to now, transformational approaches could not deal with such programs. Similar to related approaches based on separation logic 4 5 10 11 28 32, our technique relies on suitable predicates describing properties of the heap. Like [28, but in contrast to several previous works, our technique derives these heap predicates automatically from the input program and it works automatically for arbitrary data structures (i.e., not only for lists). We integrated this new technique in our fully automated termination analysis and made the resulting termination tool available via a web interface [1]. This tool automatically proves termination of Java programs on possibly cyclic data, i.e., the user does not have to provide loop preconditions, invariants, annotations, or any other manual pre-processing.

Our technique works in two steps: first, a JBC program is transformed into a termination graph, which is a finite representation of all program runs. This graph takes all sharing effects into account. Afterwards, a TRS is generated from the graph. In a similar way, we also developed techniques to analyze termination of other languages like Haskell [21] or Prolog [29] via a translation to TRSs.

Of course, one could also transform termination graphs into other formalisms than TRSs. For example, by fixing the translation from objects to integers, one could easily generate integer transition systems from the termination graph. Then the contributions of the current paper can be used as a general pre-processing approach to handle cyclic objects, which could be coupled with other termination tools. However, for methods whose termination does not rely on cyclic data, our technique is able to transform data objects into terms. For such methods, the power of existing tools for TRSs allows us to find more complex termination arguments automatically. By integrating the contributions of the current paper into our TRS-based framework, the resulting tool combines the new approach for cyclic data with the existing TRS-based approach for non-cyclic data.

In Sect. 2,4, we consider three typical classes of algorithms which rely on data that could be cyclic. The first class are algorithms where the cyclicity is irrelevant for termination. So for termination, one only has to inspect a non-cyclic part of the objects. For example, consider a doubly-linked list where the predecessor of the first and the successor of the last element are null. Here, a traversal only following the next field obviously terminates. To handle such algorithms,

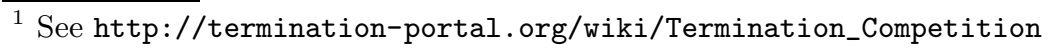


in Sect. 2 we recapitulate our termination graph framework and present a new improvement to detect irrelevant cyclicity automatically.

The second class are algorithms that mark every visited element in a cyclic object and terminate when reaching an already marked element. In Sect. 3. we develop a technique based on SMT solving to detect such marking algorithms by analyzing the termination graph and to prove their termination automatically.

The third class are algorithms that terminate because an element in a cyclic object is guaranteed to be visited a second time (i.e., the algorithms terminate when reaching a specified sentinel element). In Sect. 4, we extend termination graphs by representing definite sharing effects. Thus, we can now express that by following some field of an object, one eventually reaches another specific object. In this way, we can also prove termination of well-known algorithms like the in-place reversal for pan-handle lists [10] automatically.

We implemented all our contributions in the tool AProVE. Sect. 5 shows their applicability by an evaluation on a large benchmark collection (including numerous standard Java library programs, many of which operate on cyclic data). In our experiments, we observed that the three considered classes of algorithms capture a large portion of typical programs on cyclic data. For the treatment of (general classes of) other programs, we refer to our earlier papers [6 7 25. Moreover, in [8] we presented a technique that uses termination graphs to also detect nontermination. By integrating the new contributions of the current paper into our approach, our tool can now automatically prove termination for programs that contain methods operating on cyclic data as well as other methods operating on non-cyclic data. For the proofs of the theorems as well as all formal definitions needed for the construction of termination graphs, we refer to [9].

\section{Handling Irrelevant Cycles}

We restrict ourselves to programs without method calls, arrays, exception handlers, static fields, floating point numbers, class initializers, reflection, and multithreading to ease the presentation. However, our implementation supports these features, except reflection and multithreading. For further details, see 6 678].

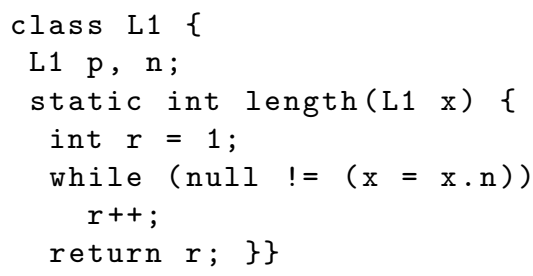

Fig. 1. Java Program

In Fig. 1, L1 is a class for (doubly-linked) lists where $\mathrm{n}$ and $\mathrm{p}$ point to the next and previous element. For brevity, we omitted a field for the value of elements. The

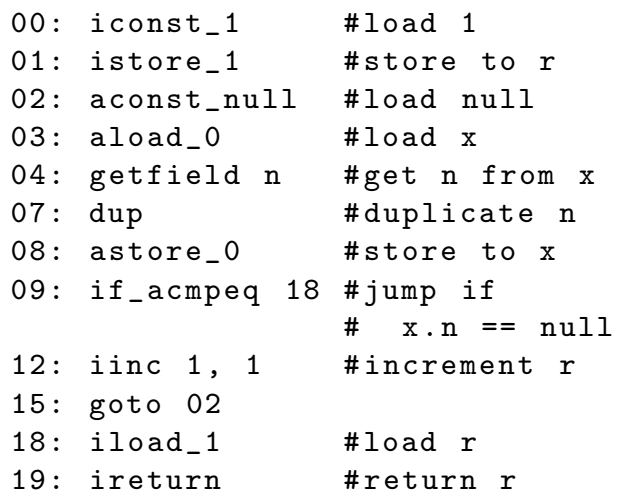

Fig. 2. JBC for length 
method length initializes a variable $r$ for the result and traverses the list until $\mathrm{x}$ is null. Fig. 2 shows the corresponding JBC obtained by the Java compiler.

After introducing program states in Sect. 2.1, we explain how termination graphs are generated in Sect. 2.2. Sect. 2.3 shows the transformation from termination graphs to TRSs. While this two-step transformation was already presented in our earlier papers, here we extend it by an improved handling of cyclic objects in order to prove termination of algorithms like length automatically.

\subsection{Abstract States in Termination Graphs}

$00\left|\mathrm{x}: o_{1}\right| \varepsilon+$ We generate a graph of abstract states from STATES $=$ PPOS $\times$ $\overline{o_{1}: \mathrm{L} 1(?)} o_{1} \circlearrowleft_{\{\mathrm{p}, \mathrm{n}\}}$ LOCVAR $\times$ OPSTACK $\times$ HEAP $\times$ ANNOTATIONS, where PPOS Fig. 3. State $A$ is the set of all program positions. Fig. 3 depicts the initial state for the method length. The first three components of a state are in the first line, separated by "|". The first component is the program position, indicated by the index of the next instruction. The second component represents the local variables as a list of references, i.e., LoCVAR $=$ REFS*2 To ease readability, in examples we denote local variables by names instead of numbers. So "x : $o_{1}$ " indicates that the 0 -th local variable $\mathrm{x}$ has the value $o_{1}$. The third component is the operand stack OPSTACK $=$ REFs* for temporary results of JBC instructions. The empty stack is denoted by $\varepsilon$ and " $O_{1}, O_{2}$ " is a stack with top element $o_{1}$.

Below the first line, information about the heap is given by a function from HEAP $=$ REFS $\rightarrow$ INTS $\cup$ UNKNOWN $\cup$ InsTANCES $\cup\{$ null $\}$ and by a set of annotations specifying sharing effects in parts of the heap that are not explicitly represented. For integers, we abstract from the different types of bounded integers in Java and consider unbounded integers instead, i.e., we cannot handle problems related to overflows. We represent unknown integers by intervals, i.e., InTS $=\{\{x \in \mathbb{Z} \mid a \leq x \leq b\} \mid a \in \mathbb{Z} \cup\{-\infty\}, b \in \mathbb{Z} \cup\{\infty\}, a \leq$ $b\}$. For readability, we abbreviate intervals such as $(-\infty, \infty)$ by $\mathbb{Z}$ and $[1, \infty)$ by $[>0]$.

Let Classnames contain all classes and interfaces in the program. The values UNKNOWN $=$ CLASSNAMES $\times\{?\}$ denote that a reference points to an unknown object or to null. Thus, " $o_{1}: \mathrm{L} 1(?)$ " means that at address $o_{1}$, we have an instance of L1 (or of its subclasses) with unknown field values or that $o_{1}$ is null.

To represent actual objects, we use InstanCES $=$ CLASSNAMES $\times($ FIELDIDS $\rightarrow$ REFs), where FIELDIDs is the set of all field identifiers. To prevent ambiguities, in general the FIELDIDs also contain the respective class names. Thus, " $o_{2}: \mathrm{L} 1\left(\mathrm{p}=o_{3}, \mathrm{n}=o_{4}\right)$ " means that at address $o_{2}$, we have some object of type L1 whose field p contains the reference $o_{3}$ and whose field $\mathrm{n}$ contains $o_{4}$.

\footnotetext{
${ }^{2}$ To avoid a special treatment of integers (which are primitive values in JBC), we also represent them using references to the heap.
} 
In our representation, if a state contains the references $o_{1}$ and $o_{2}$, then the objects reachable from $o_{1}$ resp. $o_{2}$ are disjoint 3 and tree-shaped (and thus acyclic), unless explicitly stated otherwise. This is orthogonal to the default assumptions in separation logic, where sharing is allowed unless stated otherwise, cf. e.g. [32]. In our states, one can either express sharing directly (e.g., " $o_{1}$ : $\mathrm{L} 1\left(\mathrm{p}=o_{2}, \mathrm{n}=\right.$ $o_{1}$ )" implies that $o_{1}$ reaches $o_{2}$ and is cyclic) or use annotations to indicate (possible) sharing in parts of the heap that are not explicitly represented.

The first kind of annotation is the equality annotation $o={ }^{?} o^{\prime}$, meaning that $o$ and $o^{\prime}$ could be the same. We only use this annotation if $h(o) \in$ UNKNOWN or $h\left(o^{\prime}\right) \in$ Unknown, where $h$ is the heap of the state. The second annotation is the joinability annotation $o \downarrow o^{\prime}$, meaning that $o$ and $o^{\prime}$ possibly have a common successor. To make this precise, let $o_{1} \stackrel{f}{\rightarrow} o_{2}$ denote that the object at $o_{1}$ has a field $\mathrm{f} \in$ FIELDIDs with $o_{2}$ as its value (i.e., $h\left(o_{1}\right)=(\mathrm{Cl}, e) \in$ InstancES and $\left.e(\mathrm{f})=o_{2}\right)$. For any $\pi=\mathrm{f}_{1} \ldots \mathrm{f}_{\mathrm{n}} \in$ FIELDIDs $^{*}, o_{1} \stackrel{\pi}{\rightarrow} o_{n+1}$ denotes that there exist $o_{2}, \ldots, o_{n}$ with $o_{1} \stackrel{f_{1}}{\rightarrow} o_{2} \stackrel{f_{2}}{\rightarrow} \ldots \stackrel{f_{n}-1}{\rightarrow} o_{n} \stackrel{f_{n}}{\rightarrow} o_{n+1}$. Moreover, $o_{1} \stackrel{\varepsilon}{\rightarrow} o_{1}^{\prime}$ iff $o_{1}=o_{1}^{\prime}$. Then $o \downarrow o^{\prime}$ means that there could be some $o^{\prime \prime}$ and some $\pi$ and $\tau$ such that $o \stackrel{\pi}{\rightarrow} o^{\prime \prime} \stackrel{\tau}{\leftarrow} o^{\prime}$, where $\pi \neq \varepsilon$ or $\tau \neq \varepsilon$.

In our earlier papers 6 25] we had another annotation to denote references that may point to non-tree-shaped objects. In the translation to terms later on, all these objects were replaced by fresh variables. But in this way, one cannot prove termination of length. To maintain more information about possibly nontree-shaped objects, we now introduce two new shape annotations $o \diamond$ and $o \circlearrowleft_{F I}$ instead. The non-tree annotation $o \diamond$ means that $o$ might be not tree-shaped. More precisely, there could be a reference $o^{\prime}$ with $o \stackrel{\pi_{1}}{\rightarrow} o^{\prime}$ and $o \stackrel{\pi_{2}}{\rightarrow} o^{\prime}$ where $\pi_{1}$ is no prefix of $\pi_{2}$ and $\pi_{2}$ is no prefix of $\pi_{1}$. However, these two paths from $o$ to $o^{\prime}$ may not traverse any cycles (i.e., there are no prefixes $\tau_{1}, \tau_{2}$ of $\pi_{1}$ or of $\pi_{2}$ where $\tau_{1} \neq \tau_{2}$, but $o \stackrel{\tau_{1}}{\rightarrow} o^{\prime \prime}$ and $o \stackrel{\tau_{2}}{\rightarrow} o^{\prime \prime}$ for some $\left.o^{\prime \prime}\right)$. The cyclicity annotation $o \circlearrowleft_{F I}$ means that there could be cycles including $o$ or reachable from $o$. However, any cycle must use at least the fields in $F I \subseteq$ FIELDIDs. In other words, if $o \stackrel{\pi}{\rightarrow} o^{\prime} \stackrel{\tau}{\rightarrow} o^{\prime}$ for some $\tau \neq \varepsilon$, then $\tau$ must contain all fields from $F I$. We often write $\circlearrowleft$ instead of $\circlearrowleft_{\varnothing}$. Thus in Fig. [3, $o_{1} \circlearrowleft_{\{\mathrm{p}, \mathrm{n}\}}$ means that there may be cycles reachable from $o_{1}$ and that any such cycle contains at least one $\mathrm{n}$ and one $\mathrm{p}$ field.

\subsection{Constructing the Termination Graph}

Our goal is to prove termination of length for all doubly-linked lists without "real" cycles (i.e., there is no cycle traversing only $\mathrm{n}$ or only $\mathrm{p}$ fields). Hence, $A$ is the initial state when calling the method with such an input list 4 From $A$, the termination graph in Fig. 4 is constructed by symbolic evaluation. First, iconst_1 loads the constant 1 on the operand stack. This leads to a new state connected to $A$ by an evaluation edge (we omitted this state from Fig. 4 for

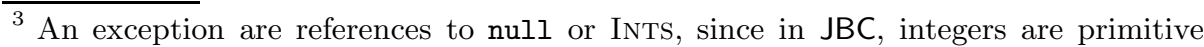
values where one cannot have any side effects. So if $h$ is the heap of a state and $h\left(o_{1}\right)=h\left(o_{2}\right) \in \operatorname{INTS}$ or $h\left(o_{1}\right)=h\left(o_{2}\right)=$ null, then one can always assume $o_{1}=o_{2}$.

4 The state $A$ is obtained automatically when generating the termination graph for a program where length is called with an arbitrary such input list, cf. Sect. 5 .
} 


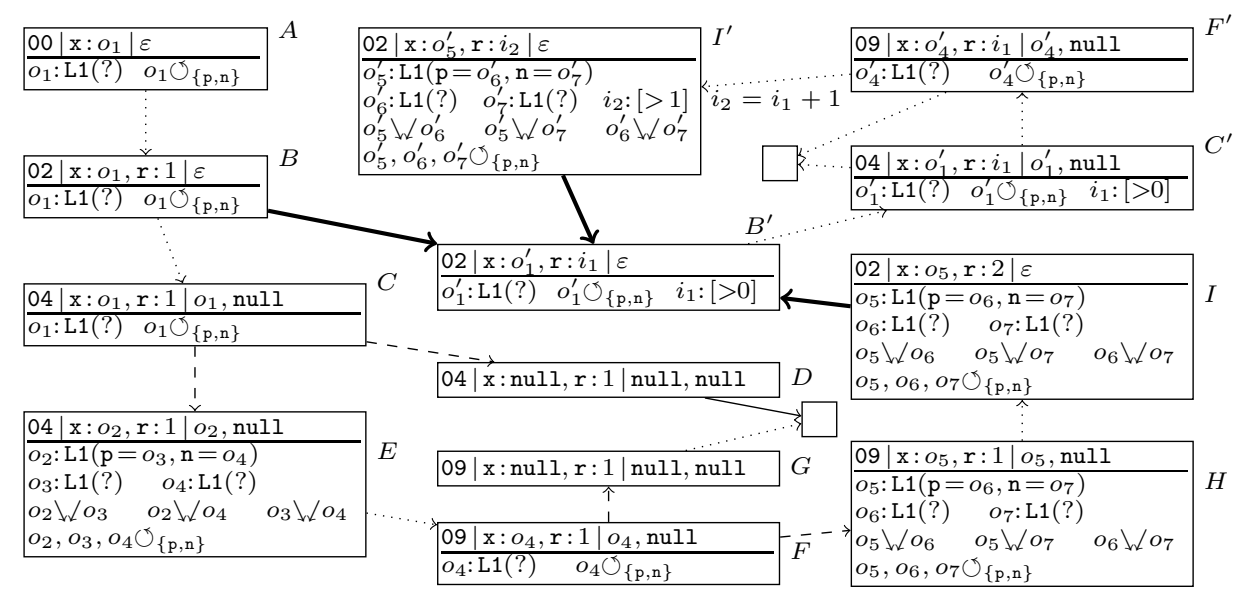

Fig. 4. Termination Graph for length

reasons of space). Then istore_1 stores the constant 1 from the top of the operand stack in the first local variable $r$. In this way, we obtain state $B$ (in Fig. 4 we use dotted edges to indicate several steps). Formally, the constant 1 is represented by some reference $i \in \operatorname{REFs}$ that is mapped to $[1,1] \in$ INTs by the heap. However, we shortened this for the presentation and just wrote $r: 1$.

In $B$, we load null and the value of $\mathrm{x}$ (i.e., $o_{1}$ ) on the operand stack, resulting in $C$. In $C$, the result of getfield depends on the value of $o_{1}$. Hence, we perform a case analysis (a so-called instance refinement) to distinguish between the possible types of $o_{1}$ (and the case where $o_{1}$ is null). So we obtain $D$ where $o_{1}$ is null, and $E$ where $o_{1}$ points to an actual object of type L1. To get single static assignments, we rename $o_{1}$ to $o_{2}$ in $E$ and create fresh references $o_{3}$ and $o_{4}$ for its fields p and $\mathrm{n}$. We connect $D$ and $E$ by dashed refinement edges to $C$.

In $E$, our annotations have to be updated. If $o_{1}$ can reach a cycle, then this could also hold for its successors. Thus, we copy $\circlearrowleft_{\{\mathrm{p}, \mathrm{n}\}}$ to the newly-created successors $o_{3}$ and $o_{4}$. Moreover, if $o_{2}$ ( $o_{1}$ under its new name) can reach itself, then its successors might also reach $o_{2}$ and they might also reach each other. Thus, we create $\downarrow$ annotations indicating that each of these references may share with any of the others. We do not have to create any equality annotations. The annotation $o_{2}={ }^{?} o_{3}$ (and $o_{2}={ }^{?} o_{4}$ ) is not needed because if the two were equal, they would form a cycle involving only one field, which contradicts $\circlearrowleft_{\{\mathrm{p}, \mathrm{n}\}}$. Furthermore, we do not need $o_{3}=?{ }^{?} o_{4}$, as $o_{1}$ was not marked with $\diamond$.

$D$ ends the program (by an exception), indicated by an empty box. In $E$, getfield $\mathrm{n}$ replaces $\mathrm{O}_{2}$ on the operand stack by the value $o_{4}$ of its field n, dup duplicates the entry $o_{4}$ on the stack, and astore_0 stores one of these entries in $\mathrm{x}$, resulting in $F$. We removed $o_{2}$ and $o_{3}$ which are no longer used in local variables or the operand stack. To evaluate if_acmpeq in $F$, we branch depending on the equality of the two top references on the stack. So we need an instance refinement and create $G$ where $o_{4}$ is null, and $H$ where $o_{4}$ refers to an actual object. The annotations in $H$ are constructed from $F$ just as $E$ was constructed from $C$. 
$G$ results in a program end. In $H$, r's value is incremented to 2 and we jump back to instruction 02 , resulting in $I$. We could continue symbolic evaluation, but this would not yield a finite termination graph. Whenever two states like $B$ and $I$ are at the same program position, we use generalization (or widening [14] ) to find a common representative $B^{\prime}$ of both $B$ and $I$. By suitable heuristics, our automation ensures that one always reaches a finite termination graph after finitely many generalization steps [8]. The values for references in $B^{\prime}$ include all values that were possible in $B$ or $I$. Since $r$ had the value 1 in $B$ and 2 in $I$, this is generalized to the interval $[>0]$ in $B^{\prime}$. Similarly, since $\mathrm{x}$ was UnKNown in $B$ but a non-null list in $I$, this is generalized to an UNKNOWN value in $B^{\prime}$.

We draw instance edges (depicted by thick arrows) from $B$ and $I$ to $B^{\prime}$, indicating that all concrete (i.e., non-abstract) program states represented by $B$ or $I$ are also represented by $B^{\prime}$. So $B$ and $I$ are instances of $B^{\prime}$ (written $B \sqsubseteq B^{\prime}$, $\left.I \sqsubseteq B^{\prime}\right)$ and any evaluation starting in $B$ or $I$ could start in $B^{\prime}$ as well.

From $B^{\prime}$ on, symbolic evaluation yields analogous states as when starting in $B$. The only difference is that now, $r$ 's value is an unknown positive integer. Thus, we reach $I^{\prime}$, where $r^{\prime}$ 's value $i_{2}$ is the incremented value of $i_{1}$ and the edge from $F^{\prime}$ to $I^{\prime}$ is labeled with " $i_{2}=i_{1}+1$ " to indicate this relation. Such labels are used in Sect. 2.3 when generating TRSs from termination graphs. The state $I^{\prime}$ is similar to $I$, and it is again represented by $B^{\prime}$. Thus, we can draw an instance edge from $I^{\prime}$ to $B^{\prime}$ to "close" the graph, leaving only program ends as leaves.

A sequence of concrete states $c_{1}, c_{2}, \ldots$ is a computation path if $c_{i+1}$ is obtained from $c_{i}$ by standard JBC evaluation. A computation sequence is represented by a termination graph if there is a path $s_{1}^{1}, \ldots, s_{1}^{k_{1}}, s_{2}^{1}, \ldots, s_{2}^{k_{2}}, \ldots$ of states in the termination graph such that $c_{i} \sqsubseteq s_{i}^{1}, \ldots, c_{i} \sqsubseteq s_{i}^{k_{i}}$ for all $i$ and such that all labels on the edges of the path (e.g., " $i_{2}=i_{1}+1$ ") are satisfied by the corresponding values in the concrete states. Thm. 1 shows that if a concrete state $c_{1}$ is an instance of some state $s_{1}$ in the termination graph, then every computation path starting in $c_{1}$ is represented by the termination graph. Thus, every infinite computation path starting in $c_{1}$ corresponds to a cycle in the termination graph.

Theorem 1 (Soundness of Termination Graphs). Let $G$ be a termination graph, $s_{1}$ some state in $G$, and $c_{1}$ some concrete state with $c_{1} \sqsubseteq s_{1}$. Then any computation sequence $c_{1}, c_{2}, \ldots$ is represented by $G$.

\subsection{Proving Termination via Term Rewriting}

From the termination graph, one can generate a TRS with built-in integers [18] that only terminates if the original program terminates. To this end, in [25] we showed how to encode each state of a termination graph as a term and each edge as a rewrite rule. We now extend this encoding to the new annotations $\diamond$ and $\circlearrowleft$ in such a way that one can prove termination of algorithms like length.

To encode states, we convert the values of local variables and operand stack entries to terms. References with unknown value are converted to variables of the same name. So the reference $i_{1}$ in state $B^{\prime}$ is converted to the variable $i_{1}$.

The null reference is converted to the constant null and for objects, we use the name of their class as a function symbol. The arguments of that function 
correspond to the fields of the class. So a list $\mathrm{x}$ of type L1 where $\mathrm{x} \cdot \mathrm{p}$ and $\mathrm{x} . \mathrm{n}$ are null would be converted to the term L1(null, null) and $o_{2}$ from state $E$ would be converted to the term $\mathrm{L} 1\left(o_{3}, o_{4}\right)$ if it were not possibly cyclic.

In 25], we had to exclude objects that were not tree-shaped from this translation. Instead, accesses to such objects always yielded a fresh, unknown variable. To handle objects annotated with $\diamond$, we now use a simple unrolling when transforming them to terms. Whenever a reference is changed in the termination graph, then all its occurrences in the unrolled term are changed simultaneously in the corresponding TRS. To handle the annotation $\circlearrowleft_{F I}$, now we only encode a subset of the fields of each class when transforming objects to terms. This subset is chosen such that at least one field of $F I$ is disregarded in the term encoding 5 Hence, when only regarding the encoded fields, the data objects are acyclic and can be represented as terms. To determine which fields to drop from the encoding, we use a heuristic which tries to disregard fields without read access.

In our example, all cyclicity annotations have the form $\circlearrowleft_{\{\mathrm{p}, \mathrm{n}\}}$ and $\mathrm{p}$ is never read. Hence, we only consider the field $\mathrm{n}$ when encoding L1-objects to terms. Thus, $o_{2}$ from state $E$ would be encoded as $\mathrm{L} 1\left(o_{4}\right)$. Now any read access to $\mathrm{p}$ would have to be encoded as returning a fresh variable.

For every state we use a function with one argument for each local variable and each entry of the operand stack. So $E$ is converted to $f_{E}\left(\operatorname{L1}\left(o_{4}\right), 1, \operatorname{L} 1\left(o_{4}\right)\right.$, null).

To encode the edges of the termination graph as rules, we consider the different kinds of edges. For a chain of evaluation edges, we obtain a rule whose left-hand side is the term resulting from the first state and whose right-hand side results from the last state of the chain. So the edges from $E$ to $F$ result in

$$
\mathrm{f}_{E}\left(\mathrm{~L} 1\left(o_{4}\right), 1, \mathrm{~L} 1\left(o_{4}\right), \text { null }\right) \rightarrow \mathrm{f}_{F}\left(o_{4}, 1, o_{4}, \text { null }\right) .
$$

In term rewriting [3, a rule $\ell \rightarrow r$ can be applied to a term $t$ if there is a substitution $\sigma$ with $\ell \sigma=t^{\prime}$ for some subterm $t^{\prime}$ of $t$. The application of the rule results in a variant of $t$ where $t^{\prime}$ is replaced by $r \sigma$. For example, consider a concrete state where $\mathrm{x}$ is a list of length 2 and the program counter is 04. This state would be an instance of the abstract state $E$ and it would be encoded by the term $\mathrm{f}_{E}(\mathrm{~L} 1(\mathrm{~L} 1($ null $)), 1, \mathrm{~L} 1(\mathrm{~L} 1($ null $))$, null $)$. Now applying the rewrite rule above yields $\mathrm{f}_{F}(\mathrm{~L} 1$ (null), $1, \mathrm{~L} 1$ (null), null). In this rule, we can see the main termination argument: Between $E$ and $F$, one list element is "removed" and the list has finite length (when only regarding the $\mathrm{n}$ field). A similar rule is created for the evaluations that lead to state $F^{\prime}$, where all occurrences of 1 are replaced by $i_{1}$.

In our old approach 25, the edges from $E$ to $F$ would result in $\mathrm{f}_{E}\left(\mathrm{~L} 1\left(o_{4}\right), 1\right.$, $\mathrm{L} 1\left(o_{4}\right)$, null $) \rightarrow \mathrm{f}_{F}\left(o_{4}^{\prime}, 1, o_{4}^{\prime}\right.$, null $)$. Its right-hand side uses the fresh variable $o_{4}^{\prime}$ instead of $o_{4}$, since this was the only way to represent cyclic objects in [25]. Since $o_{4}^{\prime}$ could be instantiated by any term during rewriting, this TRS is not terminating.

For refinement edges, we use the term for the target state on both sides of the resulting rule. However, on the left-hand side, we label the outermost function

${ }^{5}$ Of course, if $F I=\varnothing$, then we still handle cyclic objects as before and represent any access to them by a fresh variable. 
symbol with the source state. So for the edge from $F$ to $H$, we have the term for $H$ on both sides of the rule, but on the left-hand side we replace $\mathrm{f}_{H}$ by $\mathrm{f}_{F}$ :

$$
\mathrm{f}_{F}\left(\mathrm{~L} 1\left(o_{7}\right), 1, \mathrm{~L} 1\left(o_{7}\right), \text { null }\right) \rightarrow \mathrm{f}_{H}\left(\mathrm{~L} 1\left(o_{7}\right), 1, \mathrm{~L} 1\left(o_{7}\right), \text { null }\right)
$$

For instance edges, we use the term for the source state on both sides of the resulting rule. However, on the right-hand side, we label the outermost function with the target state instead. So for the edge from $I$ to $B^{\prime}$, we have the term for $I$ on both sides of the rule, but on the right-hand side we replace $f_{I}$ by $f_{B^{\prime}}$ :

$$
\mathrm{f}_{I}\left(\mathrm{~L} 1\left(o_{7}\right), 2\right) \rightarrow \mathrm{f}_{B^{\prime}}\left(\mathrm{L} 1\left(o_{7}\right), 2\right)
$$

For termination, it suffices to convert just the (non-trivial) SCCs of the termination graph to TRSs. If we do this for the only $\mathrm{SCC} B^{\prime}, \ldots, I^{\prime}, \ldots, B^{\prime}$ of our graph, and then "merge" rewrite rules that can only be applied after each other [25], then we obtain one rule encoding the only possible way through the loop:

$$
\mathrm{f}_{B^{\prime}}\left(\operatorname{L1}\left(\operatorname{L1}\left(o_{7}\right)\right), i_{1}\right) \rightarrow \mathrm{f}_{B^{\prime}}\left(\mathrm{L} 1\left(o_{7}\right), i_{1}+1\right)
$$

Here, we used the information on the edges from $F^{\prime}$ to $I^{\prime}$ to replace $i_{2}$ by $i_{1}+1$. Termination of this rule is easily shown automatically by termination provers like AProVE, although the original Java program worked on cyclic objects. However, our approach automatically detects that the objects are not cyclic anymore if one uses a suitable projection that only regards certain fields of the objects.

Theorem 2 (Proving Termination of Java by TRSs). If the TRSs resulting from the SCCs of a termination graph $G$ are terminating, then $G$ does not represent any infinite computation sequence. So by Thm. 1, the original JBC program is terminating for all concrete states $c$ where $c \sqsubseteq s$ for some state $s$ in $G$.

\section{Handling Marking Algorithms on Cyclic Data}

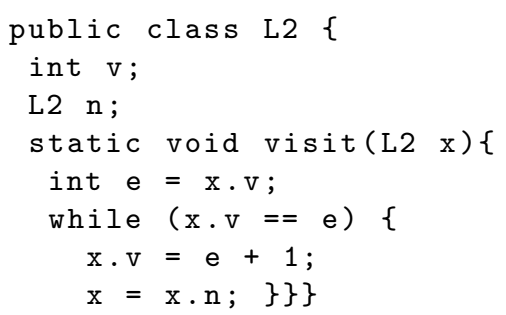

Fig. 5. Java Program

We now regard lists with a "next" field $\mathrm{n}$ where every element has an integer value $v$. The method visit stores the value of the first list element. Then it iterates over the list elements as long as they

\begin{tabular}{|c|c|c|c|}
\hline 00 : & aload_0 & & \#load $x$ \\
\hline $01:$ & getfield & $\mathrm{v}$ & \#get $v$ from $x$ \\
\hline $04:$ & istore_1 & & \#store to e \\
\hline $05:$ & aload_0 & & \#load $x$ \\
\hline $06:$ & getfield & $\mathrm{V}$ & \#get $v$ from $x$ \\
\hline $09:$ & iload_1 & & \#load e \\
\hline 10 & if_icmpne & 28 & \#jump if $x \cdot v \quad !=$ \\
\hline 13 & aload_0 & & \#load $x$ \\
\hline 14 & iload_1 & & \#load e \\
\hline 15 & iconst_1 & & \#load 1 \\
\hline 16 & iadd & & $\#$ add $e$ and 1 \\
\hline 17 & putfield & $\mathrm{V}$ & \#store to $\mathrm{x} . \mathrm{v}$ \\
\hline 20 & aload_0 & & \#load $x$ \\
\hline 21 & getfield & $\mathrm{n}$ & \#get $n$ from $x$ \\
\hline 24 & astore_0 & & \#store to $x$ \\
\hline & goto 5 & & \\
\hline & return & & \\
\hline
\end{tabular}
have the same value and "marks" them by modifying their value. If
Fig. 6. JBC for visit 


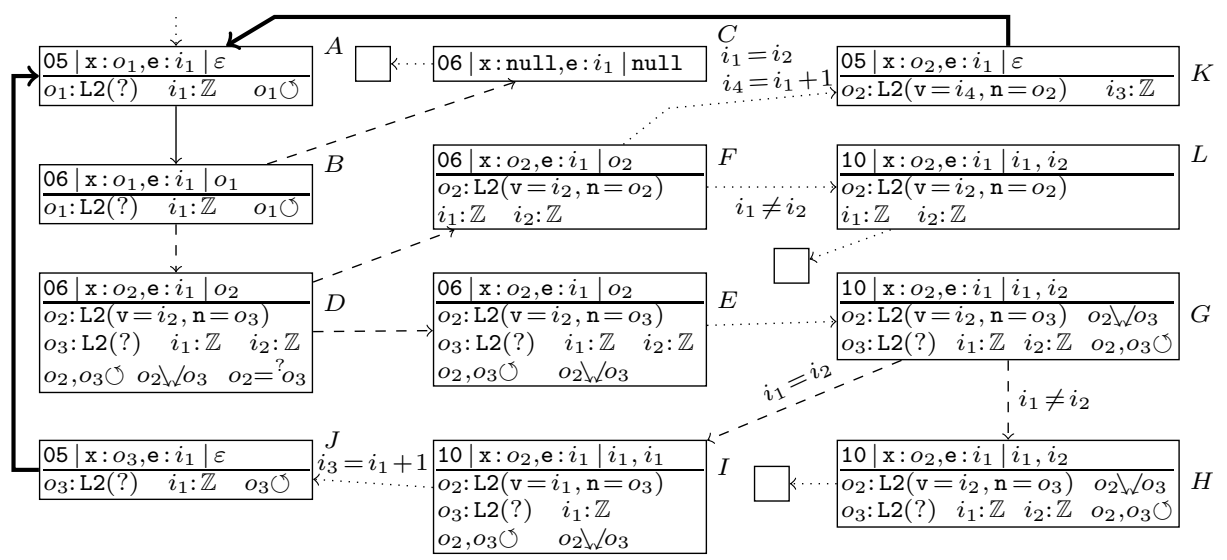

Fig. 7. Termination Graph for visit

all list elements had the same value initially, then the iteration either ends with a NullPointerException (if the list is non-cyclic) or because some element is visited for the second time (this is detected by its modified "marked" value) $6 \mathrm{We}$ illustrate the termination graph of visit in Sect. 3.1 and extend our approach in order to prove termination of such marking algorithms in Sect. 3.2.

\subsection{Constructing the Termination Graph}

When calling visit for an arbitrary (possibly cyclic) list, one reaches state $A$ in Fig. 7 after one loop iteration by symbolic evaluation and generalization. Now aload_0 loads the value $o_{1}$ of $\mathrm{x}$ on the operand stack, yielding state $B$.

To evaluate getfield $\mathrm{v}$, we perform an instance refinement and create a successor $C$ where $o_{1}$ is null and a successor $D$ where $o_{1}$ is an actual instance of L2. As in Fig. 4, we copy the cyclicity annotation to $o_{3}$ and allow $o_{2}$ and $o_{3}$ to join. Furthermore, we add $o_{2}={ }^{?} o_{3}$, since $o_{2}$ could be a cyclic one-element list.

In $C$, we end with a NullPointerException. Before accessing $o_{2}$ 's fields, we have to resolve all possible equalities. We obtain $E$ and $F$ by an equality refinement, corresponding to the cases $o_{2} \neq o_{3}$ and $o_{2}=o_{3} . F$ needs no annotations anymore, as all reachable objects are completely represented in the state.

In $E$ we evaluate getfield, retrieving the value $i_{2}$ of the field v. Then we load e's value $i_{1}$ on the operand stack, which yields $G$. To evaluate if_icmpne, we branch depending on the inequality of the top stack entries $i_{1}$ and $i_{2}$, resulting in $H$ and $I$. We label the refinement edges with the respective integer relations.

In $I$, we add 1 to $i_{1}$, creating $i_{3}$, which is written into the field $\mathrm{v}$ of $o_{2}$. Then, the field $\mathrm{n}$ of $\mathrm{O}_{2}$ is retrieved, and the obtained reference $o_{3}$ is written into $\mathrm{x}$, leading to $J$. As $J$ is a renaming of $A$, we draw an instance edge from $J$ to $A$.

\footnotetext{
${ }^{6}$ While termination of visit can also be shown by the technique of Sect. 4 which detects whether an element is visited twice, the technique of Sect.4fails for analogous marking algorithms on graphs which are easy to handle by the approach of Sect. 3 , cf. Sect. 5. So the techniques of Sect. 3 and 4 do not subsume each other.
} 
The states following $F$ are analogous, i.e., when reaching if_icmpne, we create successors depending on whether $i_{1}=i_{2}$. In that case, we reach $K$, where we have written the new value $i_{4}=i_{1}+1$ into the field $\mathrm{v}$ of $o_{2}$. Since $K$ is also an instance of $A$, this concludes the construction of the termination graph.

\subsection{Proving Termination of Marking Algorithms}

To prove termination of algorithms like visit, we try to find a suitable marking property $M \subseteq$ REFS $\times$ STATES. For every state $s$ with heap $h$, we have $(o, s) \in M$ if $o$ is reachable 7 in $s$ and if $h(o)$ is an object satisfying a certain property. We add a local variable named $c_{M}$ to each state which counts the number of references in $M$. More precisely, for each concrete state $s$ with " $c_{M}: i$ " (i.e., the value of the new variable is the reference $i), h(i) \in$ INTS is the singleton set containing the number of references $o$ with $(o, s) \in M$. For any abstract state $s$ with " $c_{M}: i$ " that represents some concrete state $s^{\prime}$ (i.e., $s^{\prime} \sqsubseteq s$ ), the interval $h(i)$ must contain an upper bound for the number of references $o$ with $\left(o, s^{\prime}\right) \in M$.

In our example, we consider the property L2.v $=i_{1}$, i.e., $c_{M}$ counts the references to L2-objects whose field $\mathrm{v}$ has value $i_{1}$. As the loop in visit only continues if there is such an object, we have $c_{M}>0$. Moreover, in each iteration, the field $\mathrm{v}$ of some L2-object is set to a value $i_{3}$ resp. $i_{4}$ which is different from $i_{1}$. Thus, $c_{M}$ decreases. We now show how to find this termination proof automatically.

To detect a suitable marking property automatically, we restrict ourselves to properties "Cl.f $\bowtie i$ ", where $\mathrm{Cl}$ is a class, $\mathrm{f}$ a field in $\mathrm{Cl}, i$ a (possibly unknown) integer, and $\bowtie$ an integer relation. Then $(o, s) \in M$ iff $h(o)$ is an object of type $\mathrm{Cl}$ (or a subtype of $\mathrm{Cl}$ ) whose field $\mathrm{f}$ stands in relation $\bowtie$ to the value $i$.

The first step is to find some integer reference $i$ that is never changed in the SCC. In our example, we can easily infer this for $i_{1}$ automatically 8

The second step is to find $\mathrm{Cl}, \mathrm{f}$, and $\bowtie$ such that every cycle of the SCC contains some state where $c_{M}>0$. We consider those states whose incoming edge has a label " $i \bowtie \ldots$ " or "...凶 $i$ ". In our example, $I$ 's incoming edge is labeled with " $i_{1}=i_{2}$ " and when comparing $i_{1}$ and $i_{2}$ in $G, i_{2}$ was the value of $\mathrm{O}_{2}$ 's field $\mathrm{v}$, where $\mathrm{O}_{2}$ is an L2-object. This suggests the marking property "L2. $\mathrm{v}$ $=i_{1}$ ". Thus, $c_{M}$ now counts the references to L2-objects whose field $\mathrm{v}$ has the value $i_{1}$. So the cycle $A, \ldots, E, \ldots A$ contains the state $I$ with $c_{M}>0$ and one can automatically detect that $A, \ldots, F, \ldots, A$ has a similar state with $c_{M}>0$.

In the third step, we add $c_{M}$ as a new local variable to all states of the SCC. For instance, in $A$ to $G$, we add " $c_{M}: i$ " to the local variables and " $i:[\geq 0]$ " to the knowledge about the heap. The edge from $G$ to $I$ is labeled with " $i>0$ " (this will be used in the resulting TRS), and in $I$ we know " $i:[>0]$ ". It remains to explain how to detect changes of $c_{M}$. To this end, we use SMT solving.

A counter for "Cl.f $\bowtie i "$ can only change when a new object of type Cl (or a subtype) is created or when the field $\mathrm{Cl} . \mathrm{f}$ is modified. So whenever "new $\mathrm{Cl}$ "

\footnotetext{
${ }^{7}$ Here, a reference $o$ is reachable in a state $s$ if $s$ has a local variable or an operand stack entry $o^{\prime}$ such that $o^{\prime} \stackrel{\pi}{\rightarrow} o$ for some $\pi \in$ FIELDIDs*.

${ }^{8}$ Due to our single static assignment syntax, this follows from the fact that at all instance edges, $i_{1}$ is matched to $i_{1}$.
} 
(or "new $\mathrm{Cl}^{\prime \prime}$ " for some subtype $\mathrm{Cl}^{\prime}$ ) is called, we have to consider the default value $d$ for the field Cl.f. If the underlying SMT solver can prove that $\neg d \bowtie i$ is a tautology, then $c_{M}$ can remain unchanged. Otherwise, to ensure that $c_{M}$ is an upper bound for the number of objects in $M, c_{M}$ is incremented by 1 .

If a putfield replaces the value $u$ in $\mathrm{Cl} . \mathrm{f}$ by $w$, we have three cases:

(i) If $u \bowtie i \wedge \neg w \bowtie i$ is a tautology, then $c_{M}$ may be decremented by 1 .

(ii) If $u \bowtie i \leftrightarrow w \bowtie i$ is a tautology, then $c_{M}$ remains the same.

(iii) In the remaining cases, we increment $c_{M}$ by 1 .

In our example, between $I$ and $J$ one writes $i_{3}$ to the field $\mathrm{v}$ of $o_{2}$. To find out how $c_{M}$ changes from $I$ to $J$, we create a formula containing all information on the edges in the path up to now (i.e., we collect this information by going backwards until we reach a state like $A$ with more than one predecessor). This results in $i_{1}=i_{2} \wedge i_{3}=i_{1}+1$. To detect whether we are in case (i) above, we check whether the information in the path implies $u \bowtie i \wedge \neg w \bowtie i$. In our example, the previous value $u$ of $o_{2} \cdot \mathrm{v}$ is $i_{1}$ and the new value $w$ is $i_{3}$. Any SMT solver for integer arithmetic can easily prove that the resulting formula

$$
i_{1}=i_{2} \wedge i_{3}=i_{1}+1 \rightarrow i_{1}=i_{1} \wedge \neg i_{3}=i_{1}
$$

is a tautology (i.e., its negation is unsatisfiable). Thus, $c_{M}$ is decremented by 1 in the step from $I$ to $J$. Since in $I$, we had " $c_{M}: i$ " with " $i:[>0]$ ", in $J$ we have " $c_{M}: i^{\prime \prime}$ " with " $i^{\prime}:[\geq 0]$ ". Moreover, we label the edge from $I$ to $J$ with the relation " $i^{\prime}=i-1$ " which is used when generating a TRS from the termination graph. Similarly, one can also easily prove that $c_{M}$ decreases between $F$ and $K$. Thm. 3 shows that Thm. 1 still holds when states are extended by counters $c_{M}$.

Theorem 3 (Soundness of Termination Graphs with Counters for Marking Properties). Let $G$ be a termination graph, s some state in $G$, $c_{1}$ some concrete state with $c_{1} \sqsubseteq s_{1}$, and $M$ some marking property. If we extend all concrete states $c$ with heap $h$ by an extra local variable " $c_{M}: i$ " such that $h(i)=\{|\{(o, c) \in M\}|\}$ and if we extend abstract states as described above, then any computation sequence $c_{1}, c_{2}, \ldots$ is represented by $G$.

We generate TRSs from the termination graph as before. So by Thm. 2 and 3 , termination of the TRSs still implies termination of the original Java program.

Since the new counter is an extra local variable, it results in an extra argument of the functions in the TRS. So for the cycle $A, \ldots, E, \ldots A$, after some "merging" of rules, we obtain the following TRS. Here, the first rule may only be applied under the condition $i>0$. For $A, \ldots, F, \ldots A$ we obtain similar rules.

$$
\begin{aligned}
\mathrm{f}_{A}(\ldots, i, \ldots) & \rightarrow \mathrm{f}_{I}(\ldots, i, \ldots) \mid i>0 \quad \mathrm{f}_{I}(\ldots, i, \ldots) \rightarrow \mathrm{f}_{J}(\ldots, i-1, \ldots) \\
\mathrm{f}_{J}\left(\ldots, i^{\prime}, \ldots\right) & \rightarrow \mathrm{f}_{A}\left(\ldots, i^{\prime}, \ldots\right)
\end{aligned}
$$

Termination of the resulting TRS can easily be be shown automatically by standard tools from term rewriting, which proves termination of the method visit. 


\section{Handling Algorithms with Definite Cyclicity}

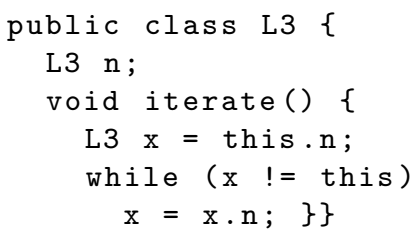

Fig. 8. Java Program

The method in Fig. 8 traverses a cyclic list until it reaches the start again. It only terminates if by following the $\mathrm{n}$ field, we reach null or the first element again. We illustrate iterate's termination graph in Sect. 4.1 and introduce a new definite reachability annotation for such algorithms. Afterwards, Sect. 4.2 shows how to prove their termination.

\subsection{Constructing the Termination Graph}

Fig. 10 shows the termination graph when calling iterate with an arbitrary list whose first element is on a cycle 9 In contrast to marking algorithms like visit in Sect. 3, iterate does not terminate for other forms of cyclic lists. State $A$ is reached after evaluating the first three instructions, where the value

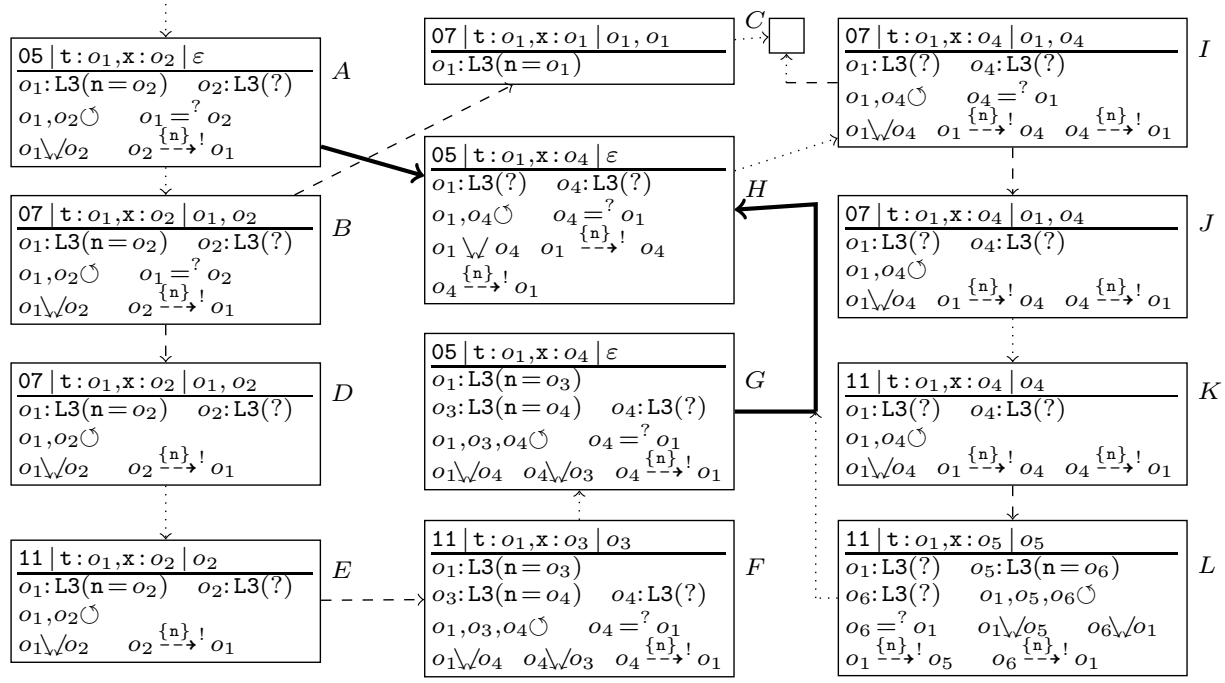

Fig. 10. Termination Graph for iterate

\footnotetext{
${ }^{9}$ The initial state of iterate's termination graph is obtained automatically when proving termination for a program where iterate is called with such lists, cf. Sect. 5 .
} 
$o_{2}$ of this.n is copied to x. In $A, o_{1}$ and $o_{2}$ are the first elements of the list, and $o_{1}=?{ }^{?} o_{2}$ allows that both are the same. Furthermore, both references are possibly cyclic and by $o_{1} \downarrow o_{2}, o_{2}$ may eventually reach $o_{1}$ again (i.e., $o_{2} \stackrel{\pi}{\rightarrow} o_{1}$ ).

Moreover, we added a new annotation $o_{2} \stackrel{\{\mathrm{n}\}}{\rightarrow} ! o_{1}$ to indicate that $o_{2}$ definitely reaches $o_{1} 11$ All previous annotations $=$ ? $, \downarrow, \diamond, \circlearrowleft$ extend the set of concrete states represented by an abstract state (by allowing more sharing). In contrast, a definite reachability annotation $o{ }_{-\rightarrow I}{ }^{\prime} o^{\prime}$ with $F I \subseteq$ FIELDIDs restricts the set of states represented by an abstract state. Now it only represents states where $o \stackrel{\pi}{\rightarrow} o^{\prime}$ holds for some $\pi \in F I^{*}$. To ensure that the $F I$-path from $o$ to $o^{\prime}$ is unique (up to cycles), FI must be deterministic. This means that for any class $\mathrm{Cl}, F I$ contains at most one of the fields of $\mathrm{Cl}$ or its superclasses. Moreover, we only use $o{ }^{F I} \rightarrow ! o^{\prime}$ if $h(o) \in$ UNKNOWN for the heap $h$ of the state.

In $A$, we load the values $o_{2}$ and $o_{1}$ of $\mathrm{x}$ and this on the stack. To evaluate if_acmpeq in $B$, we need an equality refinement w.r.t. $o_{1}={ }^{?} o_{2}$. We create $C$ for the case where $o_{1}=o_{2}$ (which ends the program) and $D$ for $o_{1} \neq o_{2}$.

In $D$, we load x's value $o_{2}$ on the stack again. To access its field $\mathrm{n}$ in $E$, we need an instance refinement for $o_{2}$. By $o_{2} \stackrel{\{\mathrm{n}\}}{\rightarrow} ! ! o_{1}, o_{2}$ 's value is not null. So there is only one successor $F$ where we replace $o_{2}$ by $o_{3}$, pointing to an L3-object. The annotation $o_{2} \stackrel{\{\mathrm{n}\}}{\rightarrow} ! o_{1}$ is moved to the value of the field $\mathrm{n}$, yielding $o_{4} \stackrel{\{\mathrm{n}\}}{\rightarrow} ! o_{1}$.

In $F$, the value $o_{4}$ of $o_{3}$ 's field $\mathrm{n}$ is loaded on the stack and written to $\mathrm{x}$. Then we jump back to instruction 05. As $G$ and $A$ are at the same program position, they are generalized to a new state $H$ which represents both $G$ and $A$. $H$ also illustrates how definite reachability annotations are generated automatically: In $A$, this reaches $\mathrm{x}$ in one step, i.e., $o_{1} \stackrel{\mathrm{n}}{\rightarrow} o_{2}$. Similarly in $G$, this reaches $\mathrm{x}$ in two steps, i.e., $o_{1} \stackrel{\mathrm{nn}}{\rightarrow} o_{4}$. To generalize this connection between this and $\mathrm{x}$ in the new state $H$ where "this : $o_{1}$ " and "x : $o_{4}$ ", one generates the annotation $o_{1} \stackrel{\{n\}}{\rightarrow} ! o_{4}$ in $H$. Thus, this definitely reaches $\mathrm{x}$ in arbitrary many steps.

From $H$, symbolic evaluation continues just as from $A$. So we reach the states $I, J, K, L$ (corresponding to $B, D, E, F$, respectively). In $L$, the value $o_{6}$ of $\mathrm{x} . \mathrm{n}$ is written to $\mathrm{x}$ and we jump back to instruction 05. There, $o_{5}$ is not referenced anymore. However, we had $o_{1} \stackrel{\{n\}}{\rightarrow} ! o_{5}$ in state $L$. When garbage collecting $o_{5}$, we "transfer" this annotation to its n-successor $o_{6}$, generating $o_{1} \stackrel{\left\{\mathbf{n}_{\rightarrow}\right.}{\rightarrow} ! o_{6}$. Now the resulting state is just a variable renaming of $H$, and thus, we can draw an instance edge to $H$. This finishes the graph construction for iterate.

\subsection{Proving Termination of Algorithms with Definite Reachability}

The method iterate terminates since the sublist between $\mathrm{x}$ and this is shortened in every loop iteration. To extract this argument automatically, we proceed similar to Sect. 3 i.e., we extend the states by suitable counters. More precisely, any state that contains a definite reachability annotation $o=-\rightarrow !$ by a counter $c_{o F-I^{\prime} o^{\prime}}$ representing the length of the $F I$-path from $o$ to $o^{\prime}$.

\footnotetext{
${ }^{10}$ In the graph, we have shortened this to $t$.

11 This annotation roughly corresponds to $l s\left(o_{2}, o_{1}\right)$ in separation logic, cf. e.g. 415].
} 
So $H$ is extended by two counters $c_{o_{1}\left\{\underline{\{n\}} \rightarrow o_{4}\right.}$ and $c_{o_{4}-\{n\} ! o_{1}}$. Information about their value can only be inferred when we perform a refinement or when we transfer an annotation $o{ }_{--\rightarrow}{ }^{\prime} o^{\prime}$ to some successor $\hat{o}$ of $o^{\prime}$ (yielding $o_{--\rightarrow}^{F I} \hat{o}$ ).

If a state $s$ contains both $o{ }_{-}^{F I} \rightarrow^{\prime} o^{\prime}$ and $o=?{ }^{?} o^{\prime}$, then an equality refinement according to $o={ }^{?} o^{\prime}$ yields two successor states. In one of them, $o$ and $o^{\prime}$ are identified and $o{ }^{F I}{ }^{\prime} o^{\prime}$ is removed. In the other successor state $s^{\prime}$ (for $o \neq o^{\prime}$ ), any path from $o$ to $o^{\prime}$ must have at least length one. Hence, if " $c_{O^{-}-\rightarrow^{\prime} O^{\prime}}: i$ " in $s$ and $s^{\prime}$, then the edge from $s$ to $s^{\prime}$ can be labeled by " $i>0$ ". So in our example, if " $c_{o_{4}\{\mathrm{n}\}}: i 0_{O_{1}} i$ " in $I$ and $J$, then we can add " $i>0$ " to the edge from $I$ to $J$.

Moreover, if $s$ contains $o{ }^{F I} \rightarrow ! o^{\prime}$ and one performs an instance refinement on $o$, then in each successor state $s^{\prime}$ of $s$, the annotation $o_{--\rightarrow}^{F I} o^{\prime}$ is replaced by $\hat{o} F_{-\rightarrow} ! o^{\prime}$ for the reference $\hat{o}$ with $o . f=\hat{o}$ where $\mathrm{f} \in F I$. Instead of " $c_{o_{-} \rightarrow{ }^{\prime} o^{\prime}}: i$ " in $s$ we now have a counter " $c_{\hat{o}^{-}-\rightarrow} o^{\prime}: i^{\prime \prime}$ " in $s^{\prime}$. Since $F I$ is deterministic, the $F I$-path from $\hat{o}$ to $o^{\prime}$ is one step shorter than the FI-path from $o$ to $o^{\prime}$. Thus, the edge from $s$ to $s^{\prime}$ is labeled by " $i^{\prime}=i-1$ ". So if we have " $c_{O_{4}-{ }^{F I} ! o_{1}}: i$ " in $K$ and " $c_{O_{6}-F^{\prime} \rightarrow o_{1}}: i^{\prime \prime}$ in $L$, then we add " $i$ ' $i-1$ " to the edge from $K$ to $L$.

When a reference $o^{\prime}$ has become unneeded in a state $s^{\prime}$ reached by evaluation from $s$, then we transfer annotations of the form $o_{--\rightarrow}^{F I} o^{\prime}$ to all successors $\hat{o}$ of $o^{\prime}$ with $o^{\prime} \stackrel{f}{\rightarrow} \hat{o}$ where $F I^{\prime}=\{f\} \cup F I$ is still deterministic. This results in a new annotation $o \underline{F I}^{\prime} !$ ! $\hat{o}$ in $s^{\prime}$. For " $c_{O^{-} I^{\prime} !}: \hat{o}$ ' $i^{\prime \prime}$ in $s^{\prime}$, we know that its value is exactly one more than " $c_{O{ }_{O-\rightarrow}{ }^{\prime}}: i$ " in $s$ and hence, we label the edge by " $i$ ' $i+1$ ". In our example, this happens between $L$ and $H$. Here the annotation $o_{1} \stackrel{\{n\}}{\rightarrow}$ ! $o_{5}$ is transferred to $o_{5}$ 's successor $o_{6}$ when $o_{5}$ is garbage collected, yielding $o_{1} \underline{\{\mathrm{n}\}} \rightarrow !$ Thm. 4 adapts Thm. 1 to definite reachability annotations.

\section{Theorem 4 (Soundness of Termination Graphs with Definite Reacha-}

bility). Let $G$ be a termination graph with definite reachability annotations, $s_{1}$ a state in $G$, and $c_{1}$ a concrete state with $c_{1} \sqsubseteq s_{1}$. As in Thm. 1, any computation sequence $c_{1}, c_{2}, \ldots$ is represented by a path $s_{1}^{1}, \ldots, s_{1}^{k_{1}}, s_{2}^{1}, \ldots, s_{2}^{k_{2}}, \ldots$ in $G$.

Let $G^{\prime}$ result from $G$ by extending the states by counters for their definite reachability annotations as above. Moreover, each concrete state $c_{j}$ in the computation sequence is extended to a concrete state $c_{j}^{\prime}$ by adding counters " $c_{O_{-\rightarrow} \rightarrow_{O^{\prime}}}: i$ " for all annotations " $O{ }_{-\rightarrow}{ }^{\prime}$ " $o$ " "in $s_{j}^{1}, \ldots, s_{j}^{k_{j}}$. Here, the heap of $c_{j}^{\prime}$ maps $i$ to the singleton interval containing the length of the FI-path between the references corresponding to o and $o^{\prime}$ in $c_{j}^{\prime}$. Then the computation sequence $c_{1}^{\prime}, c_{2}^{\prime}, \ldots$ of these extended concrete states is represented by the termination graph $G^{\prime}$.

The generation of TRSs from the termination graph works as before. Hence by Thm. 2 and 4, termination of the resulting TRSs implies that there is no infinite computation sequence $c_{1}^{\prime}, c_{2}^{\prime}, \ldots$ of extended concrete states and thus, also no infinite computation sequence $c_{1}, c_{2}, \ldots$ Hence, the Java program is terminating. Moreover, Thm. 4 can also be combined with Thm. 3, i.e., the states may also contain counters for marking properties as in Thm. 3 , 
As in Sect. 3, the new counters result in extra argument:12 of the function symbols in the TRS. In our example, we obtain the following TRS from the only SCC $I, \ldots, L, \ldots, I$ (after "merging" some rules). Termination of this TRS is easy to prove automatically, which implies termination of iterate.

$$
\begin{aligned}
\mathrm{f}_{I}(\ldots, i, \ldots) & \rightarrow \mathrm{f}_{K}(\ldots, i, \ldots) \mid i>0 \quad \mathrm{f}_{K}(\ldots, i, \ldots) \rightarrow \mathrm{f}_{L}(\ldots, i-1, \ldots) \\
\mathrm{f}_{L}\left(\ldots, i^{\prime}, \ldots\right) & \rightarrow \mathrm{f}_{I}\left(\ldots, i^{\prime}, \ldots\right)
\end{aligned}
$$

\section{Experiments and Conclusion}

We extended our earlier work 6 78 /25] on termination of Java to handle methods whose termination depends on cyclic data. We implemented our contributions in the tool AProVE [19] (using the SMT Solver Z3 [15]) and evaluated it on a collection of 387 JBC programs. It consists of al13 268 Java programs of the Termination Problem Data Base (used in the International Termination Competition); the examples length, visit, iterate from this paper 14 a variant of visit on graphs 153 well-known challenge problems from [10]; 57 (non-terminating) examples from [8]; and all 60 methods of java.util.LinkedList and java.util. HashMap from Oracle's standard Java distribution 16 Apart from list algorithms, the collection also contains many programs on integers, arrays, trees, or graphs. Below, we compare the new version of AProVE with AProVE '11 (implementing 67825, i.e., without support for cyclic data), and with the other available termination tools for Java, viz. Julia [30] and COSTA [2]. As in the Termination Competition, we allowed a runtime of 60 seconds for each example. Since the tools are tuned to succeed quickly, the results hardly change when increasing the time-out. "Yes" resp. "No" states how often termination was proved resp. disproved, "Fail" indicates failure in less than 60 seconds, "T" states how many examples led to a Time-out, and "R" gives the average Runtime in seconds for each example.

Our experiments show that AProVE is sub-

\begin{tabular}{|l||r|r|r|r|r|}
\cline { 2 - 6 } \multicolumn{1}{c||}{} & $\mathbf{Y}$ & $\mathbf{N}$ & $\mathbf{F}$ & $\mathbf{T}$ & $\mathbf{R}$ \\
\hline \hline AProVE & 267 & 81 & 11 & 28 & 9.5 \\
\hline AProVE '11 & 225 & 81 & 45 & 36 & 11.4 \\
\hline Julia & 191 & 22 & 174 & 0 & 4.7 \\
\hline COSTA & 160 & 0 & 181 & 46 & 11.0 \\
\hline
\end{tabular}
stantially more powerful than all other tools. In particular, AProVE succeeds for all problems of 10$]$ and for $85 \%$ of the examples from LinkedList and HashMap. There, AProVE '11, Julia, resp. COSTA can only handle $38 \%, 53 \%$, resp. $48 \%$. See [1] to access AProVE via a

\footnotetext{
$\overline{12 \text { For reasons }}$ of space, we only depicted the argument for the counter $o_{4} \stackrel{\{\mathrm{n}\}}{\rightarrow} ! o_{1}$.

${ }^{13}$ We removed one controversial example whose termination depends on overflows.

14 Our approach automatically infers with which input length, visit, and iterate are called, i.e., we automatically obtain the termination graphs in Fig. 44 7 and 10

${ }^{15}$ Here, the technique of Sect. 3 succeeds and the one of Sect. 4 fails, cf. Footnote 6

${ }^{16}$ Following the regulations in the Termination Competition, we excluded 7 methods from LinkedList and HashMap, as they use native methods or string manipulation.

${ }^{17}$ We are not aware of any other tool that proves termination of the algorithm for in-place reversal of pan-handle lists from 10 fully automatically.
} 
web interface, for the examples and details on the experiments, and for 6778925.

Acknowledgements. We thank F. Spoto and S. Genaim for help with the experiments and A. Rybalchenko and the anonymous referees for helpful comments.

\section{References}

1. http://aprove.informatik.rwth-aachen.de/eval/JBC-Cyclic/

2. Albert, E., Arenas, P., Codish, M., Genaim, S., Puebla, G., Zanardini, D.: Termination Analysis of Java Bytecode. In: Barthe, G., de Boer, F.S. (eds.) FMOODS 2008. LNCS, vol. 5051, pp. 2-18. Springer, Heidelberg (2008)

3. Baader, F., Nipkow, T.: Term Rewriting and All That, Cambridge (1998)

4. Berdine, J., Cook, B., Distefano, D., O'Hearn, P.W.: Automatic Termination Proofs for Programs with Shape-Shifting Heaps. In: Ball, T., Jones, R.B. (eds.) CAV 2006. LNCS, vol. 4144, pp. 386-400. Springer, Heidelberg (2006)

5. Berdine, J., Calcagno, C., Cook, B., Distefano, D., O'Hearn, P.W., Wies, T., Yang, H.: Shape Analysis for Composite Data Structures. In: Damm, W., Hermanns, H. (eds.) CAV 2007. LNCS, vol. 4590, pp. 178-192. Springer, Heidelberg (2007)

6. Brockschmidt, M., Otto, C., von Essen, C., Giesl, J.: Termination Graphs for Java Bytecode. In: Siegler, S., Wasser, N. (eds.) Walther Festschrift. LNCS, vol. 6463, pp. 17-37. Springer, Heidelberg (2010)

7. Brockschmidt, M., Otto, C., Giesl, J.: Modular termination proofs of recursive JBC programs by term rewriting. In: Proc. RTA 2011. LIPIcs, vol. 10, pp. 155-170 (2011)

8. Brockschmidt, M., Ströder, T., Otto, C., Giesl, J.: Automated detection of nontermination and NullPointerExceptions for JBC. In: Proc. FoVeOOS 2011. LNCS (2012)

9. Brockschmidt, M., Musiol, R., Otto, C., Giesl, J.: Automated termination proofs for Java programs with cyclic data. Technical Report AIB 2012-06, RWTH Aachen (2012), Available from [1] and from http://aib.informatik.rwth-aachen.de

10. Brotherston, J., Bornat, R., Calcagno, C.: Cyclic proofs of program termination in separation logic. In: Proc. POPL 2008, pp. 101-112. ACM Press (2008)

11. Cherini, R., Rearte, L., Blanco, J.: A Shape Analysis for Non-linear Data Structures. In: Cousot, R., Martel, M. (eds.) SAS 2010. LNCS, vol. 6337, pp. 201-217. Springer, Heidelberg (2010)

12. Colón, M.A., Sipma, H.B.: Practical Methods for Proving Program Termination. In: Brinksma, E., Larsen, K.G. (eds.) CAV 2002. LNCS, vol. 2404, pp. 442-454. Springer, Heidelberg (2002)

13. Cook, B., Podelski, A., Rybalchenko, A.: Termination proofs for systems code. In: Proc. PLDI 2006, pp. 415-426. ACM Press (2006)

14. Cousot, P., Cousot, R.: Abstract interpretation: A unified lattice model for static analysis of programs by construction or approximation of fixpoints. In: Proc. POPL 1977, pp. 238-252. ACM Press (1977)

15. de Moura, L., Bjørner, N.: Z3: An Efficient SMT Solver. In: Ramakrishnan, C.R., Rehof, J. (eds.) TACAS 2008. LNCS, vol. 4963, pp. 337-340. Springer, Heidelberg (2008)

16. Dershowitz, N.: Termination of rewriting. J. Symb. Comp. 3(1-2), 69-116 (1987)

17. Falke, S., Kapur, D., Sinz, C.: Termination analysis of C programs using compiler intermediate languages. In: Proc. RTA 2011. LIPIcs, vol. 10, pp. 41-50 (2011) 
18. Fuhs, C., Giesl, J., Plücker, M., Schneider-Kamp, P., Falke, S.: Proving Termination of Integer Term Rewriting. In: Treinen, R. (ed.) RTA 2009. LNCS, vol. 5595, pp. 32-47. Springer, Heidelberg (2009)

19. Giesl, J., Schneider-Kamp, P., Thiemann, R.: AProVE 1.2: Automatic Termination Proofs in the Dependency Pair Framework. In: Furbach, U., Shankar, N. (eds.) IJCAR 2006. LNCS (LNAI), vol. 4130, pp. 281-286. Springer, Heidelberg (2006)

20. Giesl, J., Thiemann, R., Schneider-Kamp, P., Falke, S.: Mechanizing and improving dependency pairs. Journal of Automated Reasoning 37(3), 155-203 (2006)

21. Giesl, J., Raffelsieper, M., Schneider-Kamp, P., Swiderski, S., Thiemann, R.: Automated termination proofs for Haskell by term rewriting. ACM TOPLAS 33(2) (2011)

22. Kroening, D., Sharygina, N., Tsitovich, A., Wintersteiger, C.M.: Termination Analysis with Compositional Transition Invariants. In: Touili, T., Cook, B., Jackson, P. (eds.) CAV 2010. LNCS, vol. 6174, pp. 89-103. Springer, Heidelberg (2010)

23. Lee, C.S., Jones, N.D., Ben-Amram, A.M.: The size-change principle for program termination. In: Proc. POPL 2001, pp. 81-92. ACM Press (2001)

24. Magill, S., Tsai, M.-H., Lee, P., Tsay, Y.-K.: Automatic numeric abstractions for heap-manipulating programs. In: Proc. POPL 2010, pp. 81-92. ACM Press (2010)

25. Otto, C., Brockschmidt, M., von Essen, C., Giesl, J.: Automated termination analysis of JBC by term rewriting. In: Proc. RTA 2010. LIPIcs, vol. 6, pp. 259-276 (2010)

26. Podelski, A., Rybalchenko, A.: A Complete Method for the Synthesis of Linear Ranking Functions. In: Steffen, B., Levi, G. (eds.) VMCAI 2004. LNCS, vol. 2937, pp. 239-251. Springer, Heidelberg (2004)

27. Podelski, A., Rybalchenko, A.: Transition invariants. In: LICS 2004, pp. 32-41 (2004)

28. Podelski, A., Rybalchenko, A., Wies, T.: Heap Assumptions on Demand. In: Gupta, A., Malik, S. (eds.) CAV 2008. LNCS, vol. 5123, pp. 314-327. Springer, Heidelberg (2008)

29. Schneider-Kamp, P., Giesl, J., Ströder, T., Serebrenik, A., Thiemann, R.: Automated termination analysis for logic programs with cut. TPLP 10(4-6), 365-381 (2010)

30. Spoto, F., Mesnard, F., Payet, É.: A termination analyser for Java Bytecode based on path-length. ACM TOPLAS 32(3) (2010)

31. Tsitovich, A., Sharygina, N., Wintersteiger, C.M., Kroening, D.: Loop Summarization and Termination Analysis. In: Abdulla, P.A., Leino, K.R.M. (eds.) TACAS 2011. LNCS, vol. 6605, pp. 81-95. Springer, Heidelberg (2011)

32. Yang, H., Lee, O., Berdine, J., Calcagno, C., Cook, B., Distefano, D., O'Hearn, P.W.: Scalable Shape Analysis for Systems Code. In: Gupta, A., Malik, S. (eds.) CAV 2008. LNCS, vol. 5123, pp. 385-398. Springer, Heidelberg (2008)

33. Zantema, H.: Termination. In: Terese (ed.) Term Rewriting Systems, pp. 181-259. Cambridge University Press (2003) 